\title{
A new surrogate modeling technique combining Kriging and polynomial chaos expansions - Application to uncertainty analysis in computational dosimetry.
}

\author{
Pierric Kersaudy ${ }^{\mathrm{a}, \mathrm{b}, \mathrm{c}, *}$, Bruno Sudret ${ }^{\mathrm{d}}$, Nadège Varsier $^{\mathrm{a}, \mathrm{b}}$, Odile Picon ${ }^{\mathrm{c}}$, Joe Wiart ${ }^{\mathrm{a}, \mathrm{b}}$ \\ a Orange Labs, 38 avenue du Général Leclerc, 92130 Issy-les-Moulineaux, France \\ ${ }^{b}$ Whist Lab, 38 avenue du Général Leclerc, 92130 Issy-les-Moulineaux, France \\ ${ }^{c}$ ESYCOM, Université Paris-Est Marne-la-Vallée, 5 boulevard Descartes, 77roo Marne-la-Vallée \\ ${ }^{d}$ ETH Zürich, Chair of Risk, Safety and Uncertainty Quantification, Stefano-Franscini-Platz 5, 8093 Zürich, Switzerland
}

\begin{abstract}
In numerical dosimetry, the recent advances in high performance computing led to a strong reduction of the required computational time to assess the specific absorption rate (SAR) characterizing the human exposure to electromagnetic waves. However, this procedure remains time-consuming and a single simulation can request several hours. As a consequence, the influence of uncertain input parameters on the SAR cannot be analyzed using crude Monte Carlo simulation. The solution presented here to perform such an analysis is surrogate modeling. This paper proposes a novel approach to build such a surrogate model from a design of experiments. Considering a sparse representation of the polynomial chaos expansions using least-angle regression as a selection algorithm to retain the most influential polynomials, this paper proposes to use the selected polynomials as regression functions for the universal Kriging model. The leave-one-out cross validation is used to select the optimal number of polynomials in the deterministic part of the Kriging model. The proposed approach, called LARS-Kriging-PC modeling, is applied to three benchmark examples and then to a full-scale metamodeling problem involving the exposure of a numerical fetus model to a femtocell device. The performances of the LARS-Kriging-PC are compared to an ordinary Kriging model and to a classical sparse polynomial chaos expansion. The LARS-Kriging-PC appears to have better performances than the two other approaches. A significant accuracy improvement is observed compared to the ordinary Kriging or to the sparse polynomial chaos depending on the studied case. This approach seems to be an optimal solution between the two other classical approaches. A global sensitivity analysis is finally performed on the LARS-Kriging-PC model of the fetus exposure problem.
\end{abstract}

Keywords: polynomial chaos expansions, Kriging, least-angle regression, dosimetry, specific absorption rate

\section{Introduction}

The wireless communications are nowadays intensively used, in spite of these heavy usages the risk perception about possible health impacts of the exposure to electromagnetic fields (EMF) is still important. Monitoring the exposure is therefore a key question for local authorities and health protection agencies, as well as manufacturers and network providers. Protection standards have been designed to ensure the compliance of wireless devices to the existing protection limits. To achieve this objective, worst case scenarios are used, but they are not suitable to assess comprehensively the real exposure induced by wireless devices that are nowadays used in versatile ways.

Over the last 20 years, large efforts have been carried out to improve dosimetry and in particular by developing computational methods. As an example, the numerical method known as Finite-Difference TimeDomain approach (FDTD), that does not requires any matrix inversion, [1] has proven efficient to calculate

*Corresponding author: pierric.kersaudy@orange.com, tel: +33145295613 
the Specific Absorption Rate (SAR) that characterizes the absorption of waves by a human body or a part of it (e.g. the brain) $[2,3,4]$. Despite the large effort carried out for the reduction of the computation time, the numerical SAR analysis remains a computationally expensive procedure. Moreover, the increasingly versatile use of wireless technologies motivates the analysis of the influence of the input parameters variability (e.g. position and type of sources) on the SAR computation. Consequently uncertainty quantification in dosimetry is a true challenge and uncertainty propagation techniques as well as computer experiments have become important topics, as in several other disciplines of engineering and natural sciences. In this paper the exposure induced by a $3 \mathrm{G}$ femtocell device is estimated for a numerical model of a pregnant woman developed in two collaborative projects called FEMONUM and FETUS [5,6]. The challenge here is to study the influence of the uncertainty on the position of the femtocell device position in the room space onto the fetus exposure.

Because computer simulation in dosimetry is time consuming, the uncertainty propagation issue cannot be addressed by a classical approach such as crude Monte Carlo simulation. Thus, more advanced statistical methods have to be resorted to. Surrogate models (a.k.a. metamodels) have emerged in the last decade as powerful statistical methods that allow one to emulate the output of a complex computational model. Of great interest here are the so-called polynomial chaos expansion [7, 8, 9] and Kriging (a.k.a. Gaussian process modeling) $[10,11,12]$.

On the one hand, the polynomial chaos (PC) theory has originally been introduced by Wiener in the case of Gaussian random input variables as the finite-dimensional Wiener polynomial chaos [13]. The output in PC theory is explicitly expressed in a suitable space with a basis constituted of multivariate Hermite polynomials that are orthogonal with respect to the joint probability measure of random input variables. This expansion was later extended to other types of random variables with different basis polynomials [14, 15] leading to the so-called generalized polynomial chaos expansions (gPCE). A major work has been achieved in the direction of the so called intrusive methods and more particularly the spectral stochastic finite elements method [7, 16] that uses a combination of the Karhunen-Loeve expansion with the finite element method (FEM) for physical systems modeled by elliptic linear boundary-value problems. As an alternative, nonintrusive methods appeared in this domain which rely upon solving a number of deterministic problems with different values of the model input vector. In this non-intrusive scope, two approaches are classically distinguished:

- The projection method $[17,18]$ that uses the orthogonality of the polynomial basis to compute the coefficients by Monte-Carlo simulation or quadrature.

- The regression method $[8,19]$ that is based on the least-square minimization of the error between the model output and its approximation.

In the regression approach, several kind of truncation schemes of the PC expansion have been studied. A classic full truncation has been studied in [20], that has the drawback to require a dramatically increasing number of model evaluations when the dimensionality (i.e. the number of input parameters in the model) increases. To circumvent this issue, some sparse representations of the truncation have been studied in $[8,9]$ in order to reduce the computational cost. Particularly, in [9], the least-angle regression (LARS) [21] algorithm is employed to keep in the truncation only the most influential polynomials. Similar sparse representations have been recently obtained using compressive sampling algorithms [22, 23]

On the other hand, the universal Kriging theory has been introduced by Matheron [24] in the field of geostatistics as a tool to interpolate discrete data considered as points of a random field trajectory. Later, this approach has been widely used in computer experiment domain [25, 10, 11, 12], sequential design of experiments [26, 27, 28] and global optimization [29]. Because of the lack of a priori knowledge about the output, the Kriging model is often used in its basic configuration known as ordinary Kriging.

It is important to point out that the research communities developing polynomial chaos expansions and Kriging models are essentially different. To our knowledge, no formal link has been established so far between these procedures. In this respect, this paper proposes to combine the least-angle regression (LARS) [21] selection algorithm used in a polynomial chaos basis [9] and universal Kriging to obtain a new family of optimized surrogate models. This approach has similarities with the so-called blind-Kriging approach 


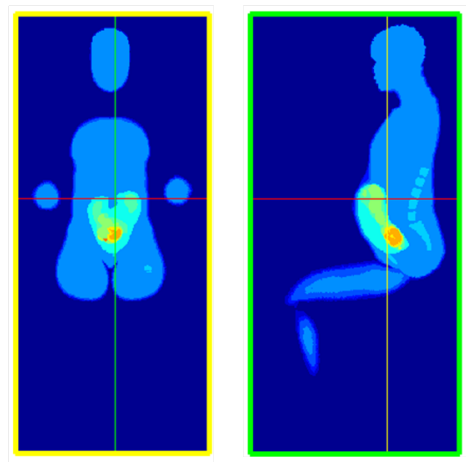

Figure 1: Numerical anatomical model of a 26-week-pregnant woman

developed in [30] but differs by the selection algorithm and the use of a polynomial chaos basis. The paper is organized as follows: in Section 2, the exposure assessment methodology is introduced, and the numerical anatomical model in which the SAR is computed is presented. In Section 3 we summarize both polynomial chaos expansion and universal Kriging approaches. In Section 4, we introduce our hybrid method which is a combination of the two previous ones. In Section 5, we apply the proposed approach to analytical examples and finally to the case study of a fetus exposure to EMF.

\section{Exposure assessment}

\subsection{Exposure quantification and anatomical models}

The human exposure is quantified by using the Specific Absorption Rate (SAR) expressed in $\mathrm{W} / \mathrm{kg}$ :

$$
S A R=\frac{\sigma E^{2}}{2 \rho}
$$

In this equation $\sigma$ is the conductivity of human tissue and $\rho$ is its mass density. $E$ is the modulus of the electric field induced in the tissue. In this paper, the exposure assessment of a pregnant woman model at the $26^{\text {th }}$ week of amenorrhea (WA) is performed. The fetus model was built from the segmentation of ante-natal images [5]. Then the obtained fetus model was inserted in a Deformable Synthetic Woman (DSW) which is initially based on a homogeneous woman model with additional basic tissues such as skin, fat, muscle and bones. This pregnant woman model was then deformed using a deformation software [31] in order to give her a seated position and the arms raised as if she was typing on a computer keyboard (see Figure 1).

\subsection{Incident Field}

Let us consider a voxelized domain $\mathcal{D} \subset \mathbb{R}^{3}$ which contains the anatomical model of the pregnant woman $\mathcal{D}_{p w}$, of the fetus $\mathcal{D}_{f}$ and the surrounding air $\mathcal{D}_{a}$, such that $\mathcal{D}_{a} \cup \mathcal{D}_{f} \cup \mathcal{D}_{p w}=\mathcal{D}$. The electromagnetic field (EMF) is computed within $\mathcal{D}$ over a time interval $[0, T]$ using the Finite-Difference Time-Domain numerical method (FDTD) which has been intensively used in the past and has shown its efficiency for the EMF computation $[1,2,3,4]$. In this study, $T=1.5 \cdot 10^{-8} s$ and the time step used in the finite-difference algorithm is $\Delta T=3.75 \cdot 10^{-12} s$ ensuring the convergence of the explicite scheme of the numerical method. To avoid spurious reflection at the boundary $\partial \mathcal{D}$ of the domain $\mathcal{D}$, a perfectly matched layer (PML) [32] is used. In this paper, the EMF source is a femtocell device. Femtocells are wireless access points offering reduced radio coverage in $3 \mathrm{G}$ and $4 \mathrm{G}$ frequency bands. These devices are designed to operate in the vicinity of users (distance of few meters). A photography of the femtocell device used in this paper and its internal view are presented in Figure 2. Basically, this source would have to be modeled inside the domain $\mathcal{D}$. However, being at a few meters distances from the anatomical model, the computation time and the memory request which 


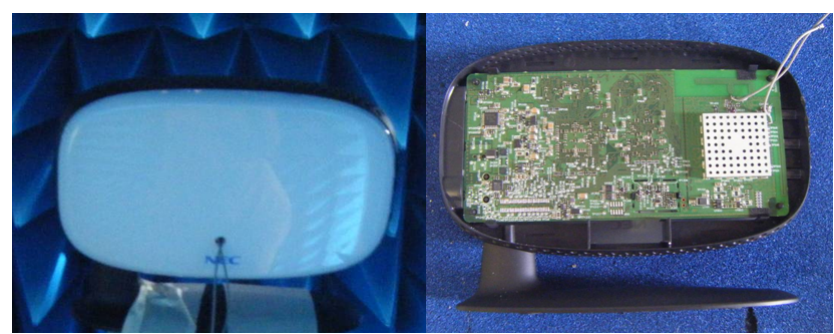

Figure 2: External and internal viexs of the considered femtocell device.

are already important may become impracticable because of the resulting huge size of $\mathcal{D}$. To circumvent this issue, let us define a domain $\mathcal{D}_{h b}$ called Huygens Box such as $\mathcal{D}_{p w} \subset \mathcal{D}_{h b} \subset \mathcal{D}$. Using the equivalence principle [33, 34], equivalent EMF sources are computed on the boundaries of the Huygens Box $\partial \mathcal{D}_{h b}$ as the result of the spherical wave theory [35] that is used to express the EMF emitted by the femtocell device in whole free space by Eq. (2) and Eq. (3):

$$
\begin{gathered}
\boldsymbol{E}(r, \theta, \phi)=\frac{k}{\sqrt{\eta}} \sum_{s=1}^{2} \sum_{n=1}^{\infty} \sum_{m=-n}^{n} Q_{s m n} \boldsymbol{F}_{s m n}(r, \theta, \phi) \\
\boldsymbol{H}(r, \theta, \phi)=-i k \sqrt{\eta} \sum_{s=1}^{2} \sum_{n=1}^{\infty} \sum_{m=-n}^{n} Q_{s m n} \boldsymbol{F}_{3-s, m, n}(r, \theta, \phi)
\end{gathered}
$$

where $\boldsymbol{E}(r, \theta, \phi)$ and $\boldsymbol{H}(r, \theta, \phi)$ are the electric and magnetic field vectors expressed in the spherical coordinates system $(r$ being the radius between the antenna and the computation point, $\theta$ the elevation angle and $\phi$ the azimut angle), $k$ and $\eta$ are respectively the propagation constant and the specific admittance, $Q_{s m n}$ and $\boldsymbol{F}_{s m n}$ are the coefficient and spherical wave function (or spherical mode) of index $s$, order $m$ and degree $n$. Spherical modes constitute an orthogonal basis of the EMF space. For a given antenna, these equations provide a formulation that fully characterizes the EMF emitted by the antenna in whole free space. In practice, the sums in Eq. (2) and Eq. (3) will always be truncated following empirical rules detailed in [35] such that the number of retained modes is large enough for ensuring convergence. Using the near-field measurements methodology described in [35], coefficients $Q_{s m n}$ have been measured for the $3 \mathrm{G}$ femtocell antenna at the frequency $2100 \mathrm{MHz}$. As explained before, with this description of the emitted $\mathrm{EMF}$, the equivalent sources of the Huygens Box $\mathcal{D}_{h b}$ may be computed through the equivalence principle $[33,34]$. Then the size of the domain $\mathcal{D}$ and thus the computational time can be considerably reduced (i.e. approximately the same size than $\mathcal{D}_{p w}$ and $\left.\mathcal{D}_{h b}\right)$. Despite the gain achieved by using this methodology, a single simulation remains time-consuming and can take up to 2 hours on a parallelized GPU architecture.

Finally, the output is the whole-fetus-body $S A R$ which is obtained by dividing the total absorbed power in $\mathcal{D}_{f}$ by the total mass of tissues within $\mathcal{D}_{f}$ :

$$
W F B S A R=\frac{P_{a b s}\left(\mathcal{D}_{f}\right)}{M\left(\mathcal{D}_{f}\right)}=\frac{\sum_{\boldsymbol{v} \in \mathcal{D}_{f}} \sigma(\boldsymbol{v}) E(\boldsymbol{v})^{2}}{2 \sum_{\boldsymbol{v} \in \mathcal{D}_{f}} \rho(\boldsymbol{v})}
$$

$\boldsymbol{v}$ representing a voxel of the domain $\mathcal{D}$, and $E(\boldsymbol{v}), \rho(\boldsymbol{v}), \sigma(\boldsymbol{v})$ being the electric-field modulus, the mass density and the conductivity computed in $\boldsymbol{v}$, respectively.

\subsection{Uncertainty propagation issues}

Assessing the fetus exposure, the challenge is to evaluate the influence of the uncertain location of the femtocell device in the space onto the whole-fetus-body SAR. Thus, four input parameters potentially influencing the output are defined (see Figure 3): 


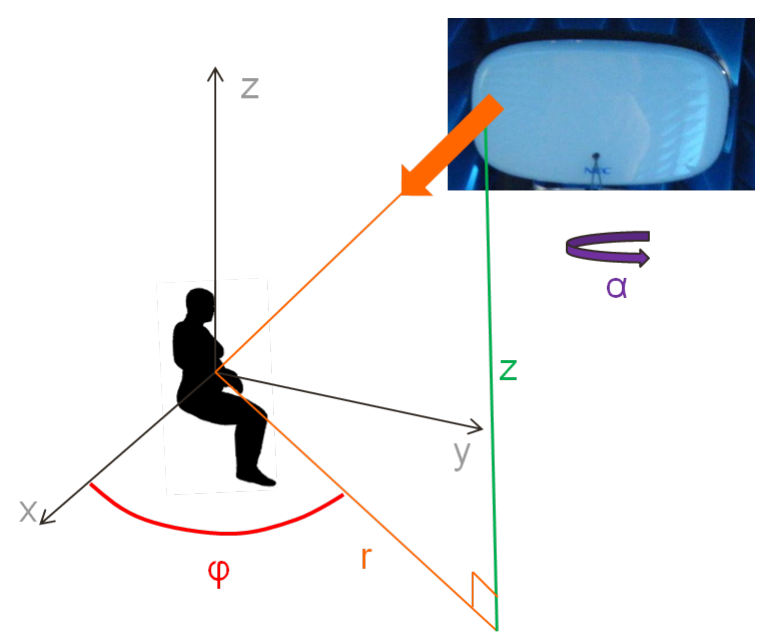

Figure 3: Geometrical input parameters for the uncertainty quantification related to the fetus exposure.

- The three cylindrical coordinates, namely $r, \phi, z$, which describe the spatial location of the antenna in a system centered on the pregnant-woman body.

- The rotation angle $\alpha$ of the antenna with respect to the vertical axis.

In this analysis, the input parameters are considered as independent. The challenge is here to propagate the uncertainty in these input parameters through the physical model Huygens Box/FDTD and to analyze the influence of the latter onto the output variable (whole-fetus-body SAR). As explained in the introduction, crude Monte Carlo simulation is impracticable to manage the uncertainty propagation issue because of the high computational cost of a single SAR simulation. Consequently, building a surrogate model for the whole fetus body SAR from a limited number of model evaluations seems to be an appropriate approach to address the uncertainty propagation issue.

\section{Surrogate modelling}

Let us consider $M$ input parameters $\boldsymbol{x}=\left\{x_{1}, \ldots, x_{M}\right\}^{T} \in \mathbb{R}^{M}$ linked to a scalar output variable $y$ (the whole body fetus SAR in the present exposure background) by the deterministic relationship $y=f(\boldsymbol{x})$ where $f$ denotes the computational model considered as a blackbox. The function $f$ here corresponds to the result of a FDTD simulation that computes the SAR depending on a particular configuration of the femtocell antenna position. Let $\mathbb{X} \subset \mathbb{R}^{M}$ be the space of the input parameters.

\subsection{Universal Kriging}

The Universal Kriging theory introduced by Matheron [24] in the field of geostatistics considers the deterministic output $y=f(\boldsymbol{x})$ as a realization of a stationary Gaussian process $Y(\boldsymbol{x})$. By denoting $Y(\boldsymbol{x}, \omega)$ a realization of the process $Y(\boldsymbol{x})$ where $\omega$ belongs to the underlying probabilistic space $\Omega$, we obtain the following formulation:

$$
Y(\boldsymbol{x}, \omega)=\sum_{k=0}^{P-1} \beta_{k} \psi_{k}(\boldsymbol{x})+Z(\boldsymbol{x}, \omega) \quad \boldsymbol{x} \in \mathbb{X}, \omega \in \Omega
$$

In the above equation $\psi=\left\{\psi_{k}, k=0 \ldots P-1\right\}$ is a collection of regression functions, $\beta=\left\{\beta_{k}, k=0 \ldots P-1\right\}$ are the regression coefficients and $Z$ is a Gaussian process $[10,11]$ indexed by $\boldsymbol{x} \in \mathbb{X}$. This Gaussian process is fully characterized by its mean value and its covariance function:

$$
E(Z(\boldsymbol{x}))=0 \quad \forall \boldsymbol{x} \in \mathbb{X}
$$




$$
C\left(\boldsymbol{x}, \boldsymbol{x}^{\prime}\right)=\sigma^{2} R\left(\boldsymbol{x}-\boldsymbol{x}^{\prime}\right) \quad \forall\left\{\boldsymbol{x}, \boldsymbol{x}^{\prime}\right\} \in \mathbb{X}^{2}
$$

Here $\sigma^{2}$ is the constant variance of the process and $R$ is the so-called autocorrelation function. In the Kriging theory, this autocorrelation function models the dependence structure between values of the stochastic process at different points and its functional form is assumed to be known. Classically, one uses componentwise anisotropic autocorrelation functions depending on the distance between two points $\boldsymbol{x}$ and $\boldsymbol{x}^{\prime} \in \mathbb{X}$, that is relevant when the input parameters have differently strong influences on the output variable. In this paper, the well-known Gaussian autocorrelation function defined in Eq. (8) is used.

$$
R\left(\boldsymbol{x}-\boldsymbol{x}^{\prime}, \boldsymbol{\theta}\right)=\exp \left(-\sum_{i=1}^{M}\left(\frac{\left|x_{i}-x_{i}^{\prime}\right|}{\theta_{i}}\right)^{2}\right)
$$

where $\boldsymbol{\theta}=\left\{\theta_{1}, \ldots, \theta_{M}\right\}$ is a vector of correlation lengths that has to be determined. Such an autocorrelation function ensures that the associated trajectories of the Gaussian process are infinitely differentiable [12], which is a suitable feature in our case, since the whole-fetus-body SAR evolution depending on spatial parameters is a very regular phenomenon.

Let us consider a design of experiments of $N$ observations $\mathcal{X}=\left\{\left(\boldsymbol{x}^{(1)}, f\left(\boldsymbol{x}^{(1)}\right)\right) \ldots\left(\mathbf{x}^{(N)}, f\left(\mathbf{x}^{(N)}\right)\right)\right\}$. Under the assumptions of the universal Kriging theory, the best linear unbiased predictor (BLUP) for the output variable $\hat{Y}(\boldsymbol{x})$ is defined by:

$$
\hat{m}_{\hat{Y}}(\boldsymbol{x})=\boldsymbol{\psi}(\boldsymbol{x})^{T} \hat{\boldsymbol{\beta}}+\boldsymbol{r}(\boldsymbol{x})^{T} \boldsymbol{R}^{-1}(\boldsymbol{y}-\boldsymbol{\Psi} \hat{\boldsymbol{\beta}})
$$

where $\boldsymbol{\psi}(\boldsymbol{x})=\left\{\psi_{0}(\boldsymbol{x}), \ldots, \psi_{P-1}(\boldsymbol{x})\right\}^{T}$ and $\hat{\boldsymbol{\beta}}=\left\{\hat{\beta}_{0}(\boldsymbol{x}), \ldots, \hat{\beta}_{P-1}(\boldsymbol{x})\right\}^{T}$ are respectively the vectors of the regression functions and estimates of the regression coefficients, $\boldsymbol{r}(\boldsymbol{x})=\left\{R\left(\boldsymbol{x}-\boldsymbol{x}^{(1)}, \boldsymbol{\theta}\right), \ldots, R\left(\boldsymbol{x}-\boldsymbol{x}^{(N)}, \boldsymbol{\theta}\right)\right\}^{T}$ is the correlation vector between $\boldsymbol{x}$ and the points of the design of experiments, $\boldsymbol{R}=\left\{R\left(\boldsymbol{x}^{(i)}-\boldsymbol{x}^{(j)}, \boldsymbol{\theta}\right)\right\}_{1<=i, j<=N}$ is the correlation matrix of the design of experiments, $\boldsymbol{y}=\left\{f\left(\boldsymbol{x}^{(1)}\right), \ldots, f\left(\boldsymbol{x}^{(N)}\right)\right\}^{T}$ is the response at the corresponding points of the experimental design and $\boldsymbol{\Psi}=\left\{\boldsymbol{\psi}\left(\boldsymbol{x}^{(1)}\right), \ldots \boldsymbol{\psi}\left(\boldsymbol{x}^{(N)}\right)\right\}^{T}$ is the matrix of the regression functions computed onto the design of experiments. In the formulation of Eq. (9), $\hat{\beta}$ is the generalized least-square estimator:

$$
\hat{\boldsymbol{\beta}}=\left(\boldsymbol{\Psi}^{T} \boldsymbol{R}^{-1} \boldsymbol{\Psi}\right)^{-1} \boldsymbol{\Psi}^{T} \boldsymbol{R}^{-1} \boldsymbol{y}
$$

Note that if the regression function ensemble is only constituted of the a constant term, the Kriging model is called Ordinary Kriging (OK). Moreover, the Kriging theory provides an uncertainty interval to this estimator of the output variable. Indeed, in Eq. (5), the model error being modeled by a Gaussian process, the BLUP is itself the mean of a Gaussian process of variance $s_{\hat{Y}}^{2}(\boldsymbol{x})$ :

$$
s_{\hat{Y}}^{2}(\boldsymbol{x})=\sigma^{2}\left(1-\boldsymbol{r}(\boldsymbol{x})^{T} \boldsymbol{R}^{-1} \boldsymbol{r}(\boldsymbol{x})+\left(\boldsymbol{F}^{T} \boldsymbol{R}^{-1} \boldsymbol{r}(\boldsymbol{x})-\boldsymbol{\psi}(\boldsymbol{x})\right)^{T}\left(\boldsymbol{\Psi}^{T} \boldsymbol{R}^{-1} \boldsymbol{\Psi}\right)^{-1}\left(\boldsymbol{F}^{T} \boldsymbol{R}^{-1} \boldsymbol{r}(\boldsymbol{x})-\boldsymbol{\psi}(\boldsymbol{x})\right)\right)
$$

The accuracy of a Kriging model is based on the accurate estimation of the hyperparameters $\boldsymbol{\theta}$ of its autocorrelation function. In this paper, the hyperparameters are estimated using the maximum likelihood approach [11]. Let us recall the likelihood expression of $\boldsymbol{y}$ with respect to its multivariate normal distribution:

$$
L\left(\boldsymbol{y} \mid \boldsymbol{\beta}, \sigma^{2}, \boldsymbol{\theta}\right)=\frac{1}{\left(\left(2 \pi \sigma^{2}\right)^{N}[\operatorname{det} \boldsymbol{R}(\boldsymbol{\theta})]\right)^{1 / 2}} \exp \left[-\frac{1}{2 \sigma^{2}}(\boldsymbol{y}-\boldsymbol{F} \boldsymbol{\beta})^{T} \boldsymbol{R}(\boldsymbol{\theta})^{-1}(\boldsymbol{y}-\boldsymbol{F} \boldsymbol{\beta})\right]
$$

One can show that the simplification of the negative $\log$-likelihood function $-\log (L)$ using the estimator of Eq. (10) leads to an expression that only depends on the hyperparameters $\boldsymbol{\theta}$ [36]. Eventually the minimization of this negative log-likelihood function (i.e. the maximization of the likelihood) is reduced to the minimization of a simpler expression called the reduced likelihood function:

$$
\psi(\boldsymbol{\theta})=\hat{\sigma^{2}}(\boldsymbol{\theta})[\operatorname{det} \boldsymbol{R}(\boldsymbol{\theta})]^{1 / N}
$$


where:

$$
\hat{\sigma^{2}}(\boldsymbol{\theta})=\frac{1}{N}[\boldsymbol{y}-\boldsymbol{\Psi} \hat{\boldsymbol{\beta}}(\boldsymbol{\theta})]^{T} \boldsymbol{R}^{-1}[\boldsymbol{\theta})(\boldsymbol{y}-\mathbf{\Psi} \hat{\boldsymbol{\beta}}(\boldsymbol{\theta})]
$$

and $N$ is the number of input points. The estimation of $\boldsymbol{\theta}$ is therefore defined by the global minimization of this reduced likelihood function. In this paper, this global optimization problem is treated with the BOXMIN algorithm of the DACE Matlab toolbox [37] which is a kind of multivariate dichotomy algorithm. It is important to notice that this optimization algorithm is quite simple, thus its performances can decrease with increasing number of input parameters $M$.

The Kriging model has several interesting properties. If the autocorrelation function is continuous, the BLUP has a prediction variance that tends to zero for all $\boldsymbol{x} \in \mathbb{X}$ when the number of observation points tends to infinity [38]. Then, the BLUP interpolates the observations at the points of the design of experiments and the output stochastic process is Gaussian at each point $\boldsymbol{x}$ conditionally to the observed points:

$$
Y(\boldsymbol{x}) \mid \boldsymbol{y} \sim \mathcal{N}\left(\hat{m}_{\hat{Y}}(\boldsymbol{x}), s_{\hat{Y}}^{2}(\boldsymbol{x})\right)
$$

However, the universal Kriging model can be difficult to build due to the lack of knowledge about relevant regression functions $\boldsymbol{\psi}(\boldsymbol{x})=\left[\psi_{0}(\boldsymbol{x}), \ldots, \psi_{P-1}(\boldsymbol{x})\right]^{T}$. In the literature [39], elaborated regression functions are used for a Kriging model when one has an a priori knowledge about the evolution of the output variable. For instance, for some cases, physical laws can provide some assumption about the shape of the output variable. But without this a priori knowledge, the preferred solution is generally to keep only a constant term and to build an ordinary Kriging model.

\subsection{Polynomial chaos expansions}

In the polynomial chaos theory, the uncertainty affecting the input vector $\boldsymbol{x}$ leads to its representation by a random vector $\boldsymbol{X}$ with prescribed probability density function (PDF) $p_{\boldsymbol{X}}(\boldsymbol{x})$ in a probability space $\left(\mathbb{R}^{M}, \mathcal{B}_{M}, \mathbb{P}_{\boldsymbol{X}}\right), \mathcal{B}_{M}$ being the Borel $\sigma$-algebra of the event space $\mathbb{R}^{M}$ and $\mathbb{P}_{\boldsymbol{X}}$ being its probability measure. Consequently, the output variable is also affected by uncertainty and is denoted by $Y=f(\boldsymbol{X})$. In the sequel, $Y$ is assumed to have finite variance and it therefore belongs to the Hilbert space $L^{2}\left(\mathbb{R}^{M}, \mathcal{B}_{M}, \mathbb{P}_{\boldsymbol{X}}\right)$ of $\mathbb{P}_{\boldsymbol{X}}$-square integrable functionals of $\boldsymbol{X}$ with respect to the inner product:

$$
E(\psi(\boldsymbol{X}) \phi(\boldsymbol{X}))=\int_{\mathbb{X}} \psi(\boldsymbol{x}) \phi(\boldsymbol{x}) p_{\boldsymbol{X}}(\boldsymbol{x}) d \boldsymbol{x}
$$

For the sake of simplicity, the input parameters are supposed to be independent, i.e.:

$$
p_{\boldsymbol{X}}(\boldsymbol{x})=\prod_{i=1}^{M} p_{X_{i}}\left(x_{i}\right)
$$

where $p_{X_{i}}\left(x_{i}\right)$ is the marginal PDF of the random input variable $X_{i}$. As $Y$ has finite variance, one can show that it can be expanded onto an orthogonal polynomial basis [16]. One method to obtain such a basis is to use multivariate polynomials. The so-called polynomial chaos expansion (PCE) is defined as follows:

$$
Y=\sum_{\boldsymbol{\alpha} \in \mathbb{N}^{M}} \beta_{\boldsymbol{\alpha}} \psi_{\boldsymbol{\alpha}}(\boldsymbol{X})
$$

Here $\boldsymbol{\alpha}=\left\{\alpha_{1} \ldots \alpha_{M}\right\}$ is the multi-index, $\beta_{\boldsymbol{\alpha}}$ 's are deterministic coefficients to be computed and $\psi_{\boldsymbol{\alpha}}$ 's are multivariate orthonormal polynomials. The independence of input parameters allows us to construct these multivariate polynomials as a tensorization of univariate orthonormal polynomials with respect to the marginal PDFs. Originally, this expansion was formulated for standard normal input parameters with Hermite polynomials $[13,7]$. However the same formulation is possible for other types of input PDFs [14] which is known as generalized PCE. For instance, in the case of uniform PDFs for input parameters in [-1,1], the orthogonal polynomials are multivariate Legendre polynomials: considering the orthonormal family of univariate 
Legendre polynomials of degree $k:\left\{L_{k}, k \in \mathbb{N}\right\}$ with respect to the uniform PDF [40], the corresponding multivariate polynomial is obtained by the following tensorization:

$$
\psi_{\boldsymbol{\alpha}}(\boldsymbol{x})=\prod_{i=1}^{M} L_{\alpha_{i}}\left(x_{i}\right)
$$

The coefficients of the expansion in Eq. (18) can be computed using stochastic Galerkin techniques [7] or so-called non-intrusive methods [41]. The projection method belongs to the latter category [17, 20]. This latter uses he property of orthogonality of the basis polynomials. The principle is to project the expansion on the subspace defined by the polynomial corresponding to the coefficient $\beta_{\boldsymbol{\alpha}}$ of interest by using the scalar product defined in Eq. (16). Estimating the $\beta_{\boldsymbol{\alpha}}$ 's boils down to an integral computation. In pratice, this approach implies a high computational cost because the integral calculation needs quadrature schemes based on Monte Carlo or quasi-Monte Carlo simulations or Gaussian quadrature rules. The second main approach of estimating the $\beta_{\boldsymbol{\alpha}}$ 's is the regression approach proposed in [19] based on early ideas by Isukapalli [42]. In this case, the principle is to truncate the expansion in Eq. (18) to a finite series with polynomials belonging to an multi-index set $\mathcal{A} \subset \mathbb{N}^{M}$ :

$$
Y=\sum_{\boldsymbol{\alpha} \in \mathcal{A}} \beta_{\boldsymbol{\alpha}} \psi_{\boldsymbol{\alpha}}(\boldsymbol{X})+\varepsilon
$$

where $\beta_{\boldsymbol{\alpha}}$ 's are approximated as proposed in Eq. (21):

$$
\left\{\hat{\beta}_{\boldsymbol{\alpha}}, \boldsymbol{\alpha} \in \mathcal{A}\right\}=\underset{\beta_{\boldsymbol{\alpha}} \in \mathbb{R}}{\arg \min } \mathbb{E}\left[\left(\sum_{\boldsymbol{\alpha} \in \mathcal{A}} \beta_{\boldsymbol{\alpha}} \psi_{\boldsymbol{\alpha}}(\boldsymbol{X})-Y\right)^{2}\right]
$$

Considering a given design of experiments of $N$ input points with corresponding output values $\mathcal{X}=$ $\left\{\left(\boldsymbol{x}^{(1)}, f\left(\boldsymbol{x}^{(1)}\right)\right) \ldots\left(\boldsymbol{x}^{(N)}, f\left(\boldsymbol{x}^{(N)}\right)\right)\right\}$ and approximating the expectation in Eq. (21) with these points, the coefficients of the $P=\operatorname{card}(\mathcal{A})$ polynomials kept in the truncation are computed using the ordinary leastsquare (OLS) estimator:

$$
\hat{\boldsymbol{\beta}}=\left(\boldsymbol{\Psi}^{T} \boldsymbol{\Psi}\right)^{-1} \boldsymbol{\Psi}^{T} \boldsymbol{y}
$$

In the latter equation $\hat{\boldsymbol{\beta}}=\left\{\hat{\beta}_{\boldsymbol{\alpha}^{(0)}}, \ldots, \hat{\beta}_{\boldsymbol{\alpha}^{(P-1)}}\right\}^{T}$ is the vector of the estimated deterministic coefficients, $\boldsymbol{\Psi}=\left\{\psi_{\boldsymbol{\alpha}^{(j)}}\left(\boldsymbol{x}^{(i)}\right)\right\}_{1 \leq i \leq N, 0 \leq j \leq P-1}$ is the matrix of polynomials computed at the design points, and $\boldsymbol{y}=$ $\left\{f\left(\boldsymbol{x}^{(1)}\right), \ldots, f\left(\boldsymbol{x}^{(N)}\right)\right\}^{T}$ is the response of the original computational model at the corresponding points. This method requires to define a level of truncation and an iterative design of experiments led by the quality of the obtained surrogate model. Indeed, for a given design of experiment of $N$ observations, the leastsquare resolution suggests that the number $P$ of polynomials kept in the truncation is lower than $N$ and must be low enough to ensure the well-conditioning of the information matrix [8]. Consequently, for a given model built from the $N$-observation design of experiments that requires more polynomials in the truncation because of its non satisfactory quality, the design of experiments size has to be increased.

Among the possible truncations, the standard one is the full $\mathrm{PC}$ truncation [20]. Considering a given degree $p$, it consists in keeping in the truncation the polynomials corresponding to the multi-index set $\mathcal{A}=\left\{\boldsymbol{\alpha} \in \mathbb{N}^{M},|\boldsymbol{\alpha}| \leq p\right\}$, where $|\boldsymbol{\alpha}|=\sum_{i=1}^{M} \alpha_{i}$ is the total degree of polynomial $\psi_{\boldsymbol{\alpha}}$. In this case, the number of polynomials in the truncation is equal to:

$$
P=\left(\begin{array}{c}
M+p \\
p
\end{array}\right)
$$

Here, $P$ increases polynomially with both the degree $p$ of the truncation and the number of input parameters $M$. Consequently, the number $N$ of simulation points in the design of experiments can quickly become impracticable for a high degree $p$. To address this problem, the truncation scheme can by optimized using the least-angle regression (LARS) method [9, 21]. This method initially developed in [21] allows one to create a truncation in which the selected polynomials are those with the most significant impact on the 
model response, i.e. the polynomials that yield the best fit of the metamodel. Consequently, this truncation provides a sparse representation of the polynomial chaos expansion (Eq. (18)). The selection of polynomials is iterative and at each step, the current model is extended depending on the correlation of polynomials with the residual. With this approach, each step of the selection method provides a new extended possible sparse basis constituted of the most influential polynomials. In the LARS theory, the output is centered and the predictors are centered and normalized over the input space. Consequently the polynomial of total degree $p=0$ (i.e. the constant term) cannot be part of the selection set. In practice this polynomial is removed from the selection set and added at the end of the LARS algorithm as the first selected polynomial. The LARS algorithm is detailed below:

1. Choose a degree $p$ of a full $\mathrm{PC}$ truncation that provides a set of $P-1$ polynomials according to Eq. (23).

2. Set the coefficients $\beta_{\boldsymbol{\alpha}^{(1)}}, \ldots, \beta_{\boldsymbol{\alpha}^{(P-1)}}$ to 0 and the initial residual vector $\boldsymbol{r}^{(0)}=\boldsymbol{y}$.

3. Select the predictor (here a polynomial) $\psi_{\boldsymbol{\alpha}^{\left(j_{1}\right)}}$ the most correlated with $\boldsymbol{y}$.

4. Move the coefficient $\beta_{\boldsymbol{\alpha}^{\left(j_{1}\right)}}$ in the direction of the predictor $\psi_{\boldsymbol{\alpha}^{\left(j_{1}\right)}}$ until the current residual $\boldsymbol{r}^{(1)}=$ $\boldsymbol{y}-\hat{\beta}_{\boldsymbol{\alpha}^{\left(j_{1}\right)}} \psi_{\boldsymbol{\alpha}^{\left(j_{1}\right)}}(\boldsymbol{\mathcal { X }})$ is equally correlated with $\psi_{\boldsymbol{\alpha}^{\left(j_{1}\right)}}$ and another predictor $\psi_{\boldsymbol{\alpha}^{\left(j_{2}\right)}}$.

5. Select this second predictor $\psi_{\boldsymbol{\alpha}^{\left(j_{2}\right)}}$ and define $\boldsymbol{u}^{(2)}$ as the unit vector bisecting $\psi_{\boldsymbol{\alpha}^{\left(j_{1}\right)}}$ and $\psi_{\boldsymbol{\alpha}^{\left(j_{2}\right)}}$.

6. For $k$ from 3 to $\min (P, N-2)$ :

(a) Move $\boldsymbol{\beta}=\left\{\beta_{\boldsymbol{\alpha}^{\left(j_{1}\right)}}, \ldots, \beta_{\boldsymbol{\alpha}^{\left(j_{k-1}\right)}}\right\}$ in the direction of $\boldsymbol{u}^{(k-1)}$ until the current residual $\boldsymbol{r}^{(k-1)}$ is equally correlated with $\boldsymbol{u}^{(k-1)}$ and another predictor $\psi_{\boldsymbol{\alpha}^{\left(j_{k}\right)}}$.

(b) Select this additional predictor $\psi_{\boldsymbol{\alpha}^{\left(j_{k}\right)}}$ and define $\boldsymbol{u}^{(k)}$ as the unit vector bisecting $\boldsymbol{u}^{(k-1)}$ and $\psi_{\boldsymbol{\alpha}^{\left(j_{k}\right)}}$.

7. Add the polynomial $\psi_{\boldsymbol{\alpha}^{(0)}}$ of total degree $p=0$ to the selected polynomials.

Consequently we obtained $L=\min (P, N-2)+1$ possible basis $\left\{\psi_{\boldsymbol{\alpha}^{(0)}}, \psi_{\boldsymbol{\alpha}^{\left(j_{1}\right)}}, \ldots, \psi_{\boldsymbol{\alpha}^{\left(j_{k}\right)}}\right\}$ with $k \in \llbracket 1, L \rrbracket$. The benefit is here to choose $P \geq N-1$ to be able to get polynomials of higher degree and build a sparse truncation at a time. Then, for each possible basis, the PC coefficients is obtained by computing the leastsquare coefficients of the selected polynomials according to the so-called LARS-OLS hybrid method [21]. The LARS algorithm has here only a selection function and is not used to estimate the PC coefficients. As shown in [21], this method always increases the empirical fit $R^{2}$. This methodology is the one employed in [9]. Note that, in contrast with universal Kriging, the polynomial chaos metamodel does not interpolate at the simulation points.

\subsection{Model validation and quality assessment}

\subsubsection{Quality assessment}

In both methods presented above, meta-models are built from designs of experiments. In such approaches, it is fundamental to assess the quality of the computed surrogate models in order to see how accurate they are. To extract a global quality from the built metamodels, the following so-called generalization error is considered:

$$
E r r=\mathbb{E}\left[(f(\boldsymbol{X})-\hat{f}(\boldsymbol{X}))^{2}\right]
$$

where $f$ is the original physical model, $\hat{f}$ is the predictor provided by the metamodel (the PC truncation or the best linear unbiased predictor provided by Kriging), $\boldsymbol{X}$ is the random vector defined in Section 3.2 and $\mathbb{E}$ is the expectation operator.

When considering universal Kriging, a classic estimator of this generalization error is the IMSE (Integrated Mean Square Error) [27, 10]:

$$
I M S E=\int_{\mathbb{X}} s_{y}^{2}(\boldsymbol{x}) p_{\boldsymbol{X}}(\boldsymbol{x}) d \boldsymbol{x}
$$


When considering polynomial chaos expansions, a first intuitive estimator is the empirical mean-square error (MSE) at the simulation points. For the following experimental design $\mathcal{X}=\left\{\left(\boldsymbol{x}^{(1)}, f\left(\boldsymbol{x}^{(1)}\right)\right) \ldots\left(\boldsymbol{x}^{(N)}, f\left(\boldsymbol{x}^{(N)}\right)\right)\right\}$ of size $N$, this empirical MSE reads:

$$
M S E=\frac{1}{N} \sum_{i=1}^{N}\left(f\left(\boldsymbol{x}^{(i)}\right)-\hat{f}\left(\boldsymbol{x}^{(i)}\right)\right)^{2}
$$

In case of designs of experiments of small size, this MSE estimator is likely to largely underestimate the actual generalization error. Moreover, these estimators can not be used for both metamodeling techniques (since Kriging is an interpolator, Eq. (26) would provide a zero value). Thus it will not be used to compare both method performances.

In this paper, in order to override the underestimation issue and to be able to compare both universal Kriging and polynomial chaos performances, the Leave-one-out cross-validation technique (LOOCV) is used. LOOCV is a frequently used method in machine learning to assess the quality of a metamodel and/or to select the best metamodel among several ones. To perform LOOCV, one point $\boldsymbol{x}^{(i)}$ is taken out of the design of experiments and the metamodel $\hat{f}^{(-i)}$ is computed from the design of experiments $\mathcal{X} \backslash\left\{\left(\boldsymbol{x}^{(i)}, f\left(\boldsymbol{x}^{(i)}\right)\right)\right\}$. Then, one can calculate the prediction error at $\boldsymbol{x}^{(i)}$ :

$$
\Delta^{(i)}=f\left(\boldsymbol{x}^{(i)}\right)-\hat{f}^{(-i)}\left(\boldsymbol{x}^{(i)}\right)
$$

After computing $\boldsymbol{\Delta}^{(i)}$ for all $\boldsymbol{x}^{(i)}$ in the design of experiments, the generalization error can be estimated by the so-called Leave-one-out error (a.k.a predicted residual sum of squares (PRESS)):

$$
\operatorname{Err}_{L O O}=\frac{1}{N} \sum_{i=1}^{N} \Delta^{(i)^{2}}
$$

Even if this estimator may be a bit optimistic compared to the actual generalization error, it is much better than a classical empirical mean-square error and it can be used to assess the generalization capacity of both universal Kriging and polynomial chaos expansions. If the $\operatorname{Err}_{L O O}$ estimator seems costly to compute, analytical expressions of this error exist for universal Kriging [43] and polynomial chaos [8] that reduce the computational cost of this cross validation. For an easier interpretation of this LOO error, a determination coefficient $Q^{2}$ can be extracted from it (which is the equivalent of the classic $R^{2}$ coefficient of the mean-square error):

$$
Q^{2}=1-\frac{\operatorname{Err}_{L O O}}{\hat{\sigma}_{y}^{2}}
$$

where $\hat{\sigma}_{y}^{2}$ is the estimated variance of the output variable. Thus, the closer $Q^{2}$ is to 1 , the better is the generalization capacity of the metamodel.

\subsubsection{Model selection}

Besides assessing the quality of the predictor, the LOOCV can be used as a model selection approach. For example, it can be used for the optimization of hyperparameters of universal Kriging autocorrelation functions instead of the maximum likelihood method [11, 44]. When considering polynomial chaos expansions, LOOCV is used to select the number of LARS polynomials on the truncation that provides the most accurate metamodel [8]. At each iteration of the LARS algorithm, a LOOCV is performed on the current truncated polynomial chaos model to evaluate its accuracy. At the last iteration of the algorithm (when all polynomials in the candidate basis have been used or after $N$ iterations, $N$ being the size of the experimental design) one selects the model with the highest $Q^{2}$ among all assessed models, i.e. the best model in terms of generalization capacity. 


\section{Combination of polynomial chaos expansions and universal Kriging}

This section introduces a surrogate modeling technique, called LARS-Kriging-PC modeling, that uses the polynomials selected by the LARS algorithm as regression functions in the universal Kriging formulation of Eq. (5). As previously explained for universal Kriging, the choice of the regression functions is often based on an a priori knowledge of the physical evolution of the output variable depending on the input parameters. Most of the time, only the constant term corresponding to the polynomial of total degree $p=0$ is kept as a regression function to make the Gaussian process evolve around the estimated mean of the output variable (ordinary Kriging). We propose here to use the generalization capacity of the polynomial chaos expansions and the ability of the LARS to select the most relevant sparse set of polynomials to build up an optimal universal Kriging approximation. The purpose of the method is to select polynomials that bring the most relevant information to the Kriging model. Thus the Gaussian process has to model the residual of the regression part. The algorithm used to build such a metamodel in this paper is the following:

1. First, build an initial design of experiments of prescribed size and define a target accuracy $t$;

2. Perform the LARS algorithm using a candidate set of polynomials;

3. Within each step of the LARS algorithm, build the universal Kriging model with the current basis of polynomials;

4. Perform a LOOCV on each built surrogate model to assess their accuracy.

5. Select the model with the highest capacity of generalization, i.e. the one yielding the highest $Q^{2}$;

6. If $Q^{2}<t$, increase the design of experiments by adding new samples and repeat the algorithm from the second step.

In this paper, the Latin Hypercube sampling (LHS) method [45] is used to build the initial design of experiments. Then, to enrich the design of experiments, the nested Latin Hypercube sampling technique is used. The principle of this technique introduced in [46] is to complement an existing LHS design in such a way that the resulting design keeps its LHS configuration, at least approximately. The benefit of the NLHS technique in the present situation is to allow one to add new samples in the initial LHS by preserving the uniformity of the LHS. Indeed the size of a LHS design has to be selected a priori because this sampling is based on a grid. The NLHS will replace the initial grid by a new one corresponding to the new total number of points. The new points will be randomly added in empty possible spaces keeping the LHS configuration of the design. For more details, see [46, 8].

Note that the first chosen LARS polynomial is always polynomial of total degree $p=0$. Thus the ordinary Kriging is the first evaluated model in the algorithm presented above. Consequently, if no polynomial bring enough relevant generalization capacity to the universal-Kriging approximation, the chosen model will be the ordinary-Kriging one.

\section{Application to analytical examples and fetus exposure}

This section is dedicated to the validation of the introduced approach. First, LARS-Kriging-PC, ordinary Kriging and classic LARS-PC are applied to three benchmark functions in order to illustrate the performances of the proposed approach compared to other surrogate modeling techniques. Then, the same techniques are applied and compared to the fetus exposure data.

As the benchmark functions are fast to evaluate, the accuracy of the metamodels in these cases will be assessed, using $N_{m}=100,000$ Monte Carlo simulation points, by the following relative mean-square error:

$$
M S E_{r}=\frac{\sum_{i=1}^{N_{m}}\left(f\left(\boldsymbol{x}^{(i)}\right)-\hat{f}\left(\boldsymbol{x}^{(i)}\right)\right)^{2}}{\sum_{i=1}^{N_{m}}\left(f\left(\boldsymbol{x}^{(i)}\right)-\hat{\mu}_{y}\right)^{2}}
$$

where $\hat{\mu}_{y}$ is the estimated mean of the output variable. As far as the application to fetus exposure is concerned, the relative error from the LOOCV will be used:

$$
\varepsilon_{r}=\frac{\operatorname{Err}_{L O O}}{\hat{\sigma}_{y}^{2}}
$$




\subsection{Sensitivity analysis}

From the obtained metamodel, a sensitivity analysis can also be conducted. Thus, the total Sobol' indices $[47,48]$ will be computed from the best linear unbiased predictor of the LARS-Kriging-PC metamodel for the fetus exposure problem in order to quantify the part of the total response variance that is explained by each of the input parameters. The Sobol' indices are computed using the so-called Sobol' decomposition of $f(\boldsymbol{x})$ :

$$
f\left(x_{1}, \ldots, x_{M}\right)=f_{0}+\sum_{i=1}^{M} f_{i}\left(x_{i}\right)+\sum_{1 \leq i<j \leq M} f_{i j}\left(x_{i}, x_{j}\right)+\ldots+f_{1,2, \ldots, M}\left(x_{1}, \ldots, x_{M}\right)
$$

In the latter equation, $f_{0}$ is a constant that corresponds to the mean of the output and each summand $f_{i_{1}, \ldots, i_{s}}\left(x_{i_{1}}, \ldots, x_{i_{s}}\right)$ has the following property:

$$
\int_{0}^{1} f_{i_{1}, \ldots, i_{s}}\left(x_{i_{1}}, \ldots, x_{i_{s}}\right) d x_{i_{k}}=0 \quad k \in \llbracket 1, s \rrbracket
$$

Eq. (33) ensures the orthogonality of the summands to each other. Considering the random input vector $\boldsymbol{X}$ of independent uniform input parameters with $X_{i} \sim \mathcal{U}(0,1)$, the so-called total variance $D$ reads:

$$
D=\operatorname{Var}(f(\boldsymbol{X}))=\int_{\mathbb{X}} f^{2}(\boldsymbol{x}) d \boldsymbol{x}-f_{0}^{2}
$$

By integrating Eq. (32), the total variance can thus be decomposed into partial variances:

$$
D=\sum_{i=1}^{M} D_{i}+\sum_{1 \leq i<j \leq M} D_{i j}+\ldots+D_{1,2, \ldots, M}
$$

Then the Sobol' indices can be defined:

$$
S_{i_{1}, \ldots, i_{s}}=\frac{D_{i_{1}, \ldots, i_{s}}}{D}
$$

The first order indices $S_{i}$ aim at giving the influence of each parameter taken alone whereas the so-called total Sobol' indices $S_{T_{i}}$ aim at assessing the total effect of an input parameter. The total indices read:

$$
S_{T_{i}}=\sum_{\mathcal{I}_{i}} D_{i_{1}, \ldots, i_{s}}
$$

where

$$
\mathcal{I}_{i}=\left\{\left(i_{1}, \ldots, i_{s}\right): \exists k, k \in \llbracket 1, s \rrbracket, i_{k}=i\right\}
$$

These total and first Sobol' indices will be computed for the fetus exposure problem using Monte Carlo simulations as detailed in [47].

\subsection{First analytical example: the Ishigami function}

The Ishigami function, widely used for benchmarking in global sensitivity analysis [49], is a function depending on three input parameters $\left\{x_{1}, x_{2}, x_{3}\right\}$ and is expressed as:

$$
f(\boldsymbol{x})=\sin x_{1}+7 \sin ^{2} x_{2}+0.1 x_{3}^{4} \sin x_{1}
$$

The input parameters are independent and have uniform distributions over $[-\pi, \pi]$. The initial LHS design of experiments consists of 40 points that are augmented by the nested LHS technique until 160 points. The classical LARS-polynomial chaos (PC), the ordinary Kriging and the new LARS-Kriging-PC approaches are applied. This analysis is replicated 50 times in order to assess the statistical uncertainty, and boxplots of the errors are shown in Figure 4. The validation error (Eq. (30)) is computed using 100,000 Monte Carlo simulation points for each surrogate modeling technique and the different numbers of points in the LHS. 

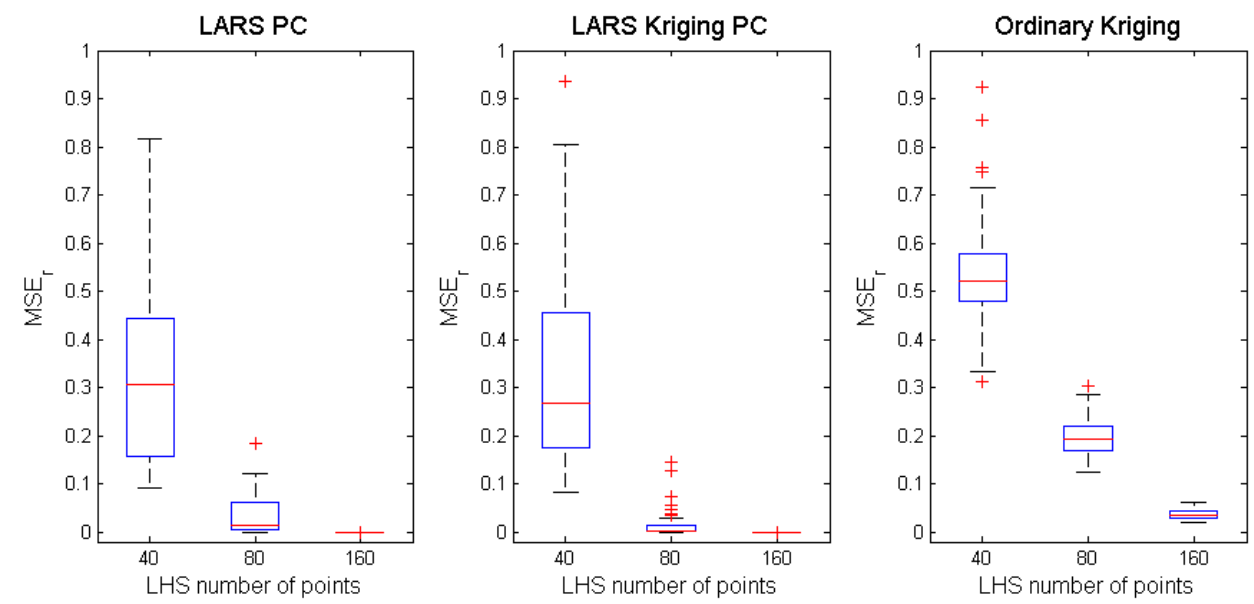

Figure 4: Comparison of LARS-PC, LARS-Kriging-PC, and ordinary Kriging metamodeling approaches for 50 different inital LHS of 40 points augmented until 160 points by the NLHS technique for the Ishigami function.

\begin{tabular}{|c|c|}
\hline Input parameter & Range \\
\hline$x_{1}$ & {$[0.05 ; 0.15]$} \\
$x_{2}$ & {$[100 ; 50000]$} \\
$x_{3}$ & {$[63070 ; 115600]$} \\
$x_{4}$ & {$[990 ; 1110]$} \\
$x_{5}$ & {$[63.1 ; 116]$} \\
$x_{6}$ & {$[700 ; 820]$} \\
$x_{7}$ & {$[1120 ; 1680]$} \\
$x_{8}$ & {$[9855 ; 12045]$} \\
\hline
\end{tabular}

Table 1: Range of the input parameters of the Borehole function

Figure 4 shows the results with boxplots presenting the median error, the quartile error values and the extreme error values of the 50 independent runs. As shown in this figure, the proposed LARS-Kriging-PC approach performs better, in terms of median value, than the two other approaches. The performance difference is apparently more significant between LARS-Kriging-PC and ordinary Kriging than between LARS-Kriging-PC and LARS-PC. However, for a small sample size (i.e. 40 points) the ordinary Kriging seems to be the least scattered method in terms of performance.

\subsection{Second analytical example: the Borehole function}

The Borehole function is a benchmark function used for emulation and prediction tests [50, 51]. This function has 8 input parameters and initially models the water flow through a borehole by the equation:

$$
f(\boldsymbol{x})=\frac{2 \pi x_{3}\left(x_{4}-x_{6}\right)}{\ln \left(x_{2} / x_{1}\right)\left(1+\frac{2 x_{7} x_{3}}{\ln \left(x_{2} / x_{1}\right) x_{1}^{2} x_{8}}+\frac{x_{3}}{x_{5}}\right)}
$$

All input parameters are modeled by independent uniform variables whose range is given in Table 1 . The same study as for the Ishigami function is conducted to illustrate the performance of the proposed approach in for this second function. Results are presented in Figure 5.

As in the previous example, the proposed LARS-Kriging-PC approach performs better than the two other techniques in terms of median value. However, for 50 points, it can be noticed that several values 

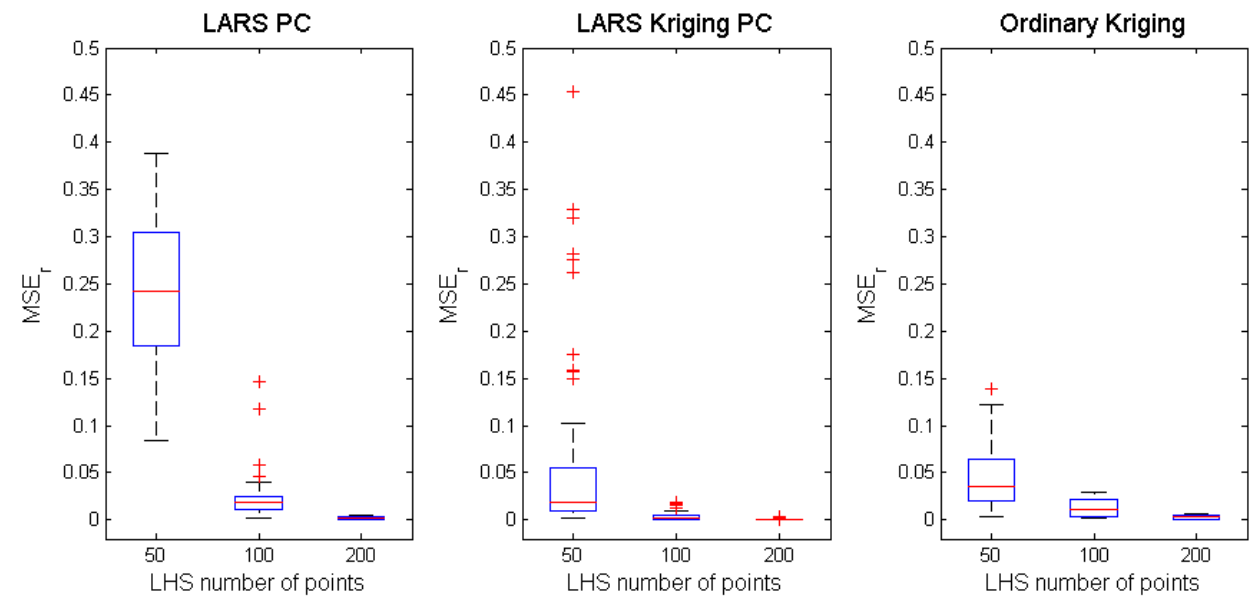

Figure 5: Comparison of LARS-PC, LARS-Kriging-PC, and ordinary Kriging metamodeling approaches for 50 different inital LHS of 50 points augmented until 200 points by the NLHS technique for the Borehole function.

considered as outliers by the boxplot routine provide higher errors than the ordinary Kriging. This can be due to a bad optimization of the hyperparameters of the universal Kriging model. Indeed, the DACE toolbox used in this study [37] provides a basic optimization algorithm that can generate suboptimal solutions in high dimension. Another explanation may be that these high errors are due to an overestimation of the generalization capacity of the LARS-Kriging-PC by the LOOCV selection. This would select a suboptimal set of functions for the deterministic part of the universal Kriging. This phenomenon is not encountered for a higher number of points where the LARS-Kriging-PC is always more efficient than the two other techniques. The prediction improvement seems to be more important for the smaller number of points when comparing LARS-Kriging-PC and LARS-PC. As in the Ishigami function, the ordinary Kriging seems to be the technique, the performance of which is the least scattered throughout the runs.

\subsection{Higher dimension example: the Sobol function}

The performance of the proposed approach is now tested with a higher dimension problem. The considered benchmark example is here the Sobol function of dimension 20 whose the expression is:

$$
f(\boldsymbol{x})=\prod_{i=1}^{20} \frac{\left|4 x_{i}-2\right|+c_{i}}{1+c_{i}} \quad \boldsymbol{x} \in[0,1]^{20}
$$

where

$$
c_{i}=\{1,2,5,10,20,50,100,500,500,500,500,500,500,500,500,500,500,500,500,500\}
$$

The same study as in the two above analytical examples is conducted for this higher dimension function. The results are shown in Figure 6. These results confirm the optimal aspect of the LARS-Kriging-PC methodology. Indeed the LARS-Kriging-PC significantly overperforms the ordinary Kriging and have at least the same performance than the classical LARS-PC. The sparsity of the Sobol function, that has a few variables more influential than the others, appears to be well captured by the LARS algorithm. Consequently the Kriging model achieves better performances than the ordinary Kriging. Furthermore, for 400 points, some cases of LARS-Kriging-PC shown as outliers appear to perform better than the two other approaches. It appears in this paper that the performances of the LARS-Kriging-PC are dependent on the problem. With a problem where LARS-PC and ordinary Kriging have poor performances, the LARS-Kriging-PC might have also poor performances and this lack of accuracy would be corrected by the addition of new points. But from an applicative point of view, the use of this approach ensures to have optimal performances regarding 

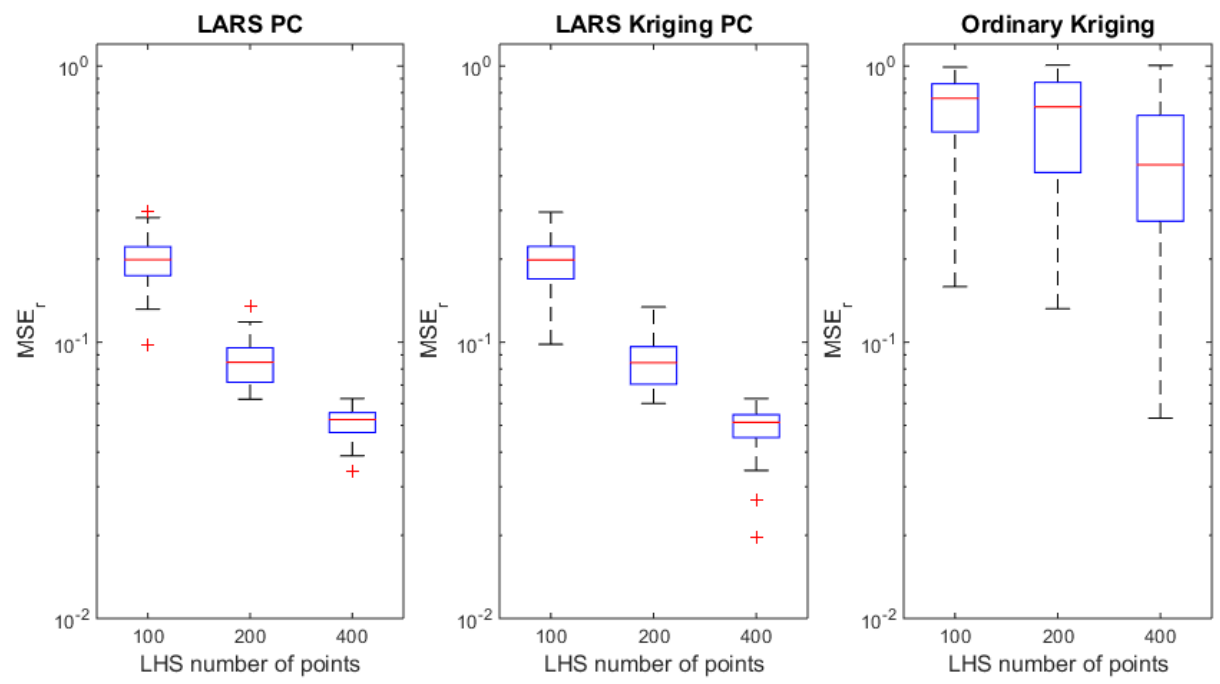

Figure 6: Comparison of LARS-PC, LARS-Kriging-PC, and ordinary Kriging metamodeling approaches for 50 different inital LHS of 100 points augmented until 400 points by the NLHS technique for the Sobol function.

\begin{tabular}{|c|c|}
\hline Input parameter & Range \\
\hline$r$ & {$[1 ; 3]$ meters } \\
$\phi$ & {$[0,360]$ degrees } \\
$z$ & {$[-0.75,1.75]$ meters } \\
$\alpha$ & {$[0,360]$ degrees } \\
\hline
\end{tabular}

Table 2: Range of the input parameters of the fetus-exposure problem

the two other methods. And because of the fact that ordinary Kriging and LARS-PC are two quite different meta-modeling techniques that might have different performances depending on the problem, the optimality of the LARS-Kriging-PC is a strong advantage allowing the user to obtain better performances or at least the best of the two.

\subsection{Application to the Fetus exposure problem}

The LARS-Kriging-PC is now applied to the fetus-exposure problem defined in Section 2. The purpose is to assess the influence of the input parameters defined in Section 2.3 on the output variable under consideration, namely the whole fetus-body SAR defined in Eq. (4). The EMF source which is a femtocell device is located in a room. Then we assume that, inside the perimeter of the room, all relative positions of the anatomical model and the femtocell device have equal chance to occur. Consequently, the 4 input parameters are assumed to follow uniform PDFs whose the ranges are listed in Table 2. These parameters are assumed to be independent. The origin of the coordinate system is located at the center of the FDTD domain. The initial design of experiments consists of a LHS of 50 points that is increased with the NLHS technique by 50 and 100 until 200 points for accuracy needs. As in the previous section, the classic LARS$\mathrm{PC}$, the ordinary Kriging and the LARS-Kriging-PC are applied on these experimental designs. The relative error obtained from the LOOCV defined in Eq. 31 is computed for these models. Figure 7 presents the results and shows that the LARS-Kriging-PC performs much better than the ordinary Kriging in terms of relative error. It can also be noticed that classic LARS-PC and LARS-Kriging-PC have equivalent performances and generate relative errors around 0.05 for 100 and 200 points in the design of experiments. In order to assess the relative influence of input parameters onto the output variance of the metamodel, the total 


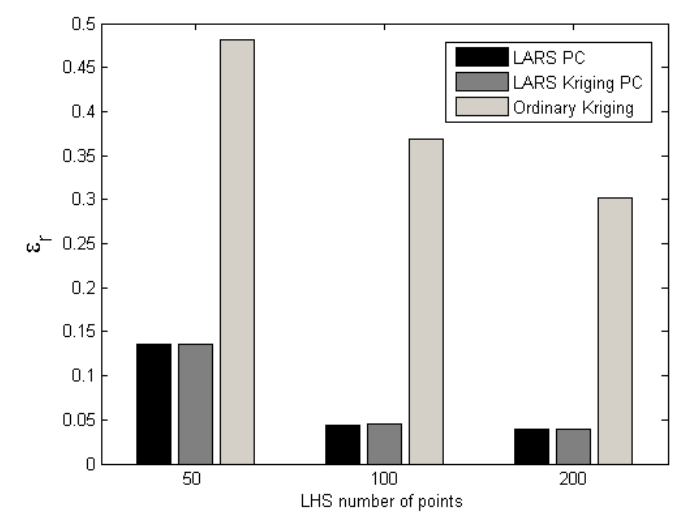

Figure 7: Comparison of LARS-PC, LARS-Kriging-PC, and ordinary Kriging metamodeling approaches on the fetus exposure problem using the relative LOO error $\varepsilon_{r}$ as a function of the number of points of the LHS

\begin{tabular}{|c|c|c|}
\hline Input parameter & First-order indice & Total indice \\
\hline$r$ & 0.171 & 0.508 \\
$\phi$ & 0.389 & 0.764 \\
$z$ & 0.014 & 0.261 \\
$\alpha$ & 0.002 & 0.131 \\
\hline
\end{tabular}

Table 3: Total and first-order Sobol indices of the input parameters of fetus exposure problem obtained from 1,000,000 MC simulations of the BLUP of the LARS-Kriging-PC metamodel.

Sobol indices [47] are estimated using Monte Carlo simulations and the LARS-Kriging-PC metamodel are computed for each input parameter using 1,000,000 Monte Carlo simulations. These total Sobol indices are presented in Table 3. It shows that the output variance is mainly explained by the input parameters $r$ and $\phi$. In particular, the most influential variable with respect to the whole-fetus-body SAR is the angle $\phi$ which is the rotation of the femtocell device around the anatomical model. This is partly explained by the fact that the thickness of pregnant woman tissues between the air and the fetus strongly varies with the rotation of the device around the pregnant woman and so the power absorbed by the fetus will also strongly vary. Moreover Table 3 shows a significant difference between first-order and total indices which means that there are strong interactions between the input parameters. The computation of the higher-order indices shows that for the second order indices, only the index of the interaction between $r$ and $\phi$ is significant and the indices of order three and four are the ones that constitute the output variance explained by $z$ and $\alpha$, always in interaction with $r$ and/or $\phi$.

\section{Conclusion}

This paper introduces the use of polynomial chaos expansions as regression functions in a universal Kriging model with an application to the estimation of fetus RF exposure from a femtocell using computational dosimetry. The proposed approach is compared in terms of performances with a classic sparse polynomial chaos expansion based on the least-angle regression (LARS) algorithm and with an ordinary Kriging model. The accuracy of the best linear unbiased predictors of the Kriging model and of the polynomial chaos expansions is estimated by leave-one-out cross validation. The comparison is first performed for benchmark functions in order to validate the approach (in this case, a large validation set can be used to compute the error), and then for the study of the influence of input parameters on the whole-fetus-body SAR. For the fetus-exposure study, the comparison is followed by a sensitivity analysis of the best linear unbiased predictor 
of the LARS-Kriging-PC model. Thus total Sobol indices are computed using Monte Carlo simulations.

As far as the benchmark examples are concerned, the novel LARS-Kriging-PC approach appears to perform better in terms of median value of the obtained error (the median being computed with respect to replications of the procedure) than classical LARS-PC expansion and ordinary Kriging. Depending on the case, the proposed approach provides a significant accuracy improvement compared to the ordinary Kriging for the Ishigami and the Sobol functions and the LARS-PC expansion for the Borehole function. In any case, it performs at least as well as the ordinary Kriging or LARS-PC taken separately. Regarding the fetus exposure problem, LARS-Kriging-PC appears to have the same accuracy as the LARS-PC expansion but performs much better than the ordinary Kriging model. The sensitivity analysis highlights that the most influential input parameter is the rotation of the antenna around the pregnant woman followed by the distance between the anatomical model and the antenna.

The proposed method has been applied to three benchmark functions and a full-scale metamodeling problem in order to obtain a better global accuracy of the created metamodels. In future investigations, this approach could be applied to global optimization problems and sequential design of experiments oriented to the evaluation of quantiles or oriented to reliability analysis $[27,28,52,53]$.

\section{Acknowledgements}

This work has been conducted in the framework of the French/Japanese FETUS (http://whist.instituttelecom.fr/fetus/) project financed by JST (Japan science and technology agency) and ANR (French research agency).

\section{References}

[1] A. Taflove, S. C. Hagness, Computational Electrodynamics. The Finite-Difference Time Domain Method., 3rd Edition, Artech House, Boston, 2005.

[2] E. Conil, A. Hadjem, A. Gati, M.-f. Wong, J. Wiart, Influence of Plane-Wave Incidence Angle on Whole Body and Local Exposure at $2100 \mathrm{MHz}$, IEEE Transactions on Electromagnetic Compatibility 53 (1) (2011) 48-52.

[3] E. Conil, A. Hadjem, F. Lacroux, M. F. Wong, J. Wiart, Variability analysis of SAR from $20 \mathrm{MHz}$ to $2.4 \mathrm{GHz}$ for different adult and child models using finite-difference time-domain, Physics in Medicine and Biology 53 (2008) 1511-1525.

[4] J. Wiart, A. Hadjem, M. F. Wong, I. Bloch, Analysis of RF exposure in the head tissues of children and adults, Physics in Medicine and Biology 53 (2008) 3681-3695.

[5] L. Bibin, J. Anquez, J. P. De la Plata Alcade, T. Boubekeur, E. D. Angelini, I. Bloch, Whole-Body Pregnant Woman Modeling by Digital Geometry Processing With Detailed Uterofetal Unit Based on Medical Images, IEEE Transactions on Biomedical Engineering 57 (10) (2010) 2346-2358.

[6] M. Jala, E. Conil, N. Varsier, J. Wiart, A. Hadjem, E. Moulines, C. Lévy-Leduc, Simplified pregnant woman models for the fetus exposure assessment, Compte Rendus Physique 14 (2013) 412-417.

[7] R. G. Ghanem, P. D. Spanos, Stochastic Finite Elements: A Spectral Approach, Courier Dover Publications, 2003.

[8] G. Blatman, B. Sudret, An adaptive algorithm to build up sparse polynomial chaos expansions for stochastic finite element analysis, Probabilistic Engineering Mechanics 25 (2) (2010) 183-197.

[9] G. Blatman, B. Sudret, Adaptive sparse polynomial chaos expansion based on least angle regression, Journal of Computational Physics 230 (6) (2011) 2345-2367.

[10] J. Sacks, S. B. Schiller, W. J. Welch, Design for Computer Experiments, Technometrics 31 (1) (1989) $41-47$.

[11] C. E. Rasmussen, C. K. I. Williams, Gaussian processes for machine learning., MIT Press, Cambridge, 2006.

[12] T. Santner, B. Williams, W. Notz, The design and analysis of computer experiments. Springer, Springer Series in Statistics, 2003.

[13] N. Wiener, The Homogeneous Chaos, American Journal of Mathematics 60 (4) (1938) 897-936.

[14] D. Xiu, G. E. M. Karniadakis, The Wiener-Askey polynomial chaos for stochastic differential equations, SIAM Journal on Scientific Computing 24 (2) (2002) 619-644.

[15] D. Lucor, G. Karniadakis, Physical Systems with Random Uncertainties: Chaos Representations with Arbitrary Probability Measure, SIAM Journal on Scientific Computing 26 (2) (2004) 750-735.

[16] C. Soize, R. Ghanem, Physical Systems with Random Uncertainties: Chaos Representations with Arbitrary Probability Measure, SIAM Journal on Scientific Computing 26 (2) (2004) 395-410.

[17] O. P. Le Maitre, M. T. Reagan, H. N. Najm, R. G. Ghanem, O. M. Knio, A Stochastic Projection Method for Fluid Flow, Journal of Computational Physics 181 (1) (2002) 9-44.

[18] L. Gilli, D. Lathouwers, J. Kloosterman, T. van der Hagen, a.J. Koning, D. Rochman, Uncertainty quantification for criticality problems using non-intrusive and adaptive Polynomial Chaos techniques, Annals of Nuclear Energy 56 (2013) $71-80$. 
[19] M. Berveiller, B. Sudret, M. Lemaitre, Stochastic finite elements: a non intrusive approach by regression, Eur. J. Comput. Mech. 15 (1-3) (2006) 81-92.

[20] G. Blatman, B. Sudret, Sparse polynomial chaos expansions and adaptive stochastic finite elements using a regression approach, Comptes Rendus Mécanique 336 (6) (2008) 518-523.

[21] B. Efron, T. Hastie, I. Johnstone, R. Tibshirani, LEAST ANGLE REGRESSION, The Annals of Statistics 32 (2) (2004) 407-499.

[22] A. Doostan, H. Owhadi, A non-adapted sparse approximation of PDEs with stochastic inputs, Journal of Computational Physics 230 (8) (2011) 3015-3034.

[23] J. Peng, J. Hampton, A. Doostan, A weighted 11-minimization approach for sparse polynomial chaos expansions, Journal of Computational Physics 267 (15) (2014) 92-111.

[24] G. Matheron, Les cahiers du Centre de morphologie mathématique de Fontainebleau, Fascicule 1: Le krigeage universel, Ecole de Mines de Paris, Fontainebleau, 1969.

[25] J. Sacks, W. J. Welch, T. J. Mitchell, H. P. Wynn, Design and Analysis of Computer Experiments, Statistical Science 4 (4) (1989) 409-423.

[26] J. Bect, D. Ginsbourger, L. Li, V. Picheny, E. Vasquez, Sequential design of experiments for estimation of a probability of failure, Statistics and Computing 22 (3) (2011) 773-793.

[27] V. Picheny, D. Ginsbourger, O. Roustant, R. T. Haftka, N.-H. Kim, Adaptive Designs of Experiments for Accurate Approximation of a Target Region, Journal of Mechanical Design 132 (7) (2010) 071008.

[28] V. Dubourg, B. Sudret, J. Bourinet, Reliability-based design optimization using kriging surrogates and subset simulation, Struct. Multidisc. Optim. 44 (5) (2011) 673-690.

[29] D. Jones, M. Schonlau, W. Welch, Efficient Global Optimization of expensive black-box functions, Journal of Global Optimization 13 (4) (1998) 455-492.

[30] V. Joseph, A. Sudjianto, Y. Hung, Blind Kriging: a new method for developing metamodels, ASME Journal of Mechanical Design 130 (3) (2008) 031102-1-8.

[31] Empire xccel. URL http: //www.empire.de/

[32] J. Berenger, A perfectly matched layer for the absorption of electromagnetic waves, Journal of Computational Physics 114 (2) (1994) 185-200.

[33] D. Merewether, R. Fisher, F. Smith, On implementing a numeric huygens source scheme in a finite difference program to illuminate scattering bodies., IEEE Transactions on Nuclear Science 27 (6) (1980) 1829-1833.

[34] R. Holland, J. Williams, Total-field versus scattered field finite difference codes: a comparative assessment., IEEE Transactions on Nuclear Science 30 (6) (1983) 4583-4588.

[35] J. E. Hansen, Spherical Near-Field antenna measurements, Peter Peregrinus Ltd, London, UK, 1988.

[36] A. Marrel, B. Iooss, F. Van Dorpe, E. Volkova, An efficient methodology for modeling complex computer codes with Gaussian processes., Computaional Statistics \& Data Analysis 52 (2008) 4731-4744.

[37] S. Lophaven, H. Nielsen, J. Sondergaard, DACE, A Matlab Kriging Toolbox, Technical University of Denmark, 2002.

[38] E. Vazquez, Modélisation comportementale de systèmes non-linéaires multivariables par méthodes à noyaux et applications., Ph.D. thesis, Université Paris XI - UFR scientifique d'Orsay (2005).

[39] J. Oakley, Estimating percentiles of uncertain computer code outputs, Journal of the Royal Statistical Society: Series C (Applied Statistics) 53 (1) (2004) 83-93.

[40] M. Abramovitz, I. A. Stegun, Handbook of Mathematical Functions with Formulas, Graphs, and Mathematical Tables, Dover Publications, New-York, NY, 1972.

[41] B. Sudret, Uncertainty propagation and sensitivity analysis in mechanical models - Contributions to structural reliability and stochastic spectral methods, habilitation à diriger des recherches, Université Blaise Pascal, Clermont-Ferrand, France (2007).

[42] S. Isukapalli, Uncertainty analysis of transport-transformation models, Ph.D. thesis, The State University of New Jersey. (1999).

[43] O. Dubrule, Cross validation of kriging in a unique neighborhood, Journal of the International Association for Mathematical Geology 15 (6) (1983) 687-699.

[44] F. Bachoc, Cross Validation and Maximum Likelihood estimation of hyper-parameters of Gaussian processes with model misspecification, Computational Statistics and Data Analysis 66 (2013) 55-69.

[45] J. S. Park, Optimal Latin-Hypercube designs for computer experiments, Journal of Statistical Planning and Inference 39 (1994) 95-111.

[46] G. Wang, Adaptive response surface method using inherited latin hypercube design points, J. Mech. Des. 125 (2003) 210-220.

[47] I. M. Sobol, Global sensitivity indices for nonlinear mathematical models and their Monte Carlo estimates, Mathematics and Computers in Simulation 55 (2001) 271-280.

[48] B. Sudret, Global sensitivity analysis using polynomial chaos expansions, Reliability Engineering \& System Safety 93 (7) (2008) 964-979.

[49] A. Saltelli, K. Chan, E. M. Scott, Sensitivity analysis, J Wiley and Sons, 2000.

[50] M. Morris, T. Mitchell, D. Ylvisaker, Bayesian design and analysis of computer experiments: use of derivatives in surface prediction, Technometrics 35 (3) (1993) 243-255.

[51] S. Xiong, P. Qian, C. Wu, Sequential design and analysis of high-accuracy and low-accuracy computer codes., Technometrics 55 (1) (2013) 37-46.

[52] B. Echard, N. Gayton, M. Lemaire, AK-MCS: An active learning reliability method combining Kriging and Monte Carlo 
Simulation, Structural Safety 33 (2) (2011) 145-154.

[53] B. Bichon, J. McFarland, S. Mahadevan, Efficient surrogate models for reliability analysis of systems with multiple failure modes, Reliability Enineering \& System Safety 96 (10) (2011) 1386-1395. 